Disclosure of Interests: Yoshinobu Koyama Paid instructor for: Asahi Kasei Pharma, Speakers bureau: BMS, Chugai, Ayumi and Eli Lilly, Soichiro Fuke: None declared, Yoshiharu Sato: None declared, Akemi Senoh: None declared, Toshie Higuchi: None declared DOI: 10.1136/annrheumdis-2019-eular.6628

\section{AB0209 A STUDY OF CORRELATION BETWEEN PLATELETS AND LYMPHOCYTE SUBSETS IN SCLEROSIS}

Jie Liang, LI Zhao-Hua, Fangyuan Hu, Ning Yan, LI Xiaofeng, Caihong Wang. the Second Hospital of Shanxi Medical University, Taiyuan, China

Background: Systemic sclerosis (SSc) is an autoimmune disease charac terized by vasculopathy, autoimmunity and widespread dermal and visceral fibrosis. There are existing evidence to support excessive platelet activation and their contribution to vascular function and fibrosis. Now we will focus on the immune role of PLT in SSC.

Objectives: By analyzing the correlation between platelet and lymphocyte subsets, CD4 + T cell subsets and disease activity in patients with diffuse SSc (dcSSc)/limitedSSc (IcSSc), and to explore the immune role of PLT in SSC.

Methods: The peripheral blood of 21 stable disease patients, 31 active disease patients and 20 healthy controls $(\mathrm{HC})$ were collected. The clinical data and laboratory indicators of them were enrolled. The T, B, NK lymphocyte subsets and CD4 + T cell subsets were detected by flow cytometry (FCM). The CD4 + T cell subsets contains Th1, Th2, TH17, Treg, Th1/Th2 and Th17/Treg. Non-parametric Kruskal-Wallis $H$ test was performed on multiple independent samples. The correlation between variables was used by Spearman correlation analysis.

Results: The PLT,PCT,MPV,PDW in peripheral blood of the stable group and active group were significantly higher than the $H C \operatorname{group}(P<0.05)$. The amount of $T$ cells in peripheral blood of active group were [1127.80 (796.66,1363.79)], which was lower than the percentage of HC group [1246.44(984.81,1497.84)] and stable group [1428.59(1179.09,2022.88)], there was significant difference between active group and stable group $(Z=6.525, P=0.038)$. The amount of NK cells of the stable group [198.48 (140.22, 302.97)] and active group [143.76(89.00, 230.00)]were significantly lower than the $\mathrm{HC}$ group $[224.81(155.04,350.51)](Z=8.694, P<0.05)$. The amount of Th cells of the stable group[888.11(679.55, 1430.70)] and active group[684.20(385,878)] were higher than the HC group[574.84 $(493.22,728.00)]$, the former was significant, there was significant difference between active group and stable $\operatorname{group}(Z=8.694, \quad P<0.05)$. The Th17/Treg ratio of the stable group $[0.18(0.10,0.34)]$ and active group $[0.21(0.13,028]$ were lower than the HC group $[0.25(0.12,0.31)]$, there was significant difference between active group and $\mathrm{HC}$ group $(Z=0.508$, $\mathrm{P}<0.05)$. The ESR and CRP were positively correlated with PLT, PCT; The amount of Th cells and Th17 cells were positively correlated with PLT,PCT; The amount of Th2 cells and the ratio of CD4+T/CD8+ T were positively correlated with PCT; There was a negative correlation between the ratio of Th1/Th2 and PCT; The amount of Th2 cells was positively correlated with MVP, and the ratio of Th1/Th2 was negatively correlated with MPV.Among these immune cells, NK cells was negatively correlated with ESR and DSS(disease severity scale).

Conclusion: In scleroderma, NK cells are associated with the severity of the disease, and PLT affects the number of Th1/Th2, Th17, and disease activity.

\section{REFERENCES:}

[1] Ntelis K, Solomou E E, Sakkas L, et al. The role of platelets in autoimmunity, vasculopathy and fibrosis. Implications for systemic sclerosis[J]. Seminars in Arthritis and Rheumatism, 2017:S0049017217301105.

[2] Pauling J D, O'Donnell, V. B, Mchugh N J. The contribution of platelets to the pathogenesis of Raynaud"s phenomenon and systemic sclerosis[J]. Platelets, 2013, 24(7):503-515.

Acknowledgement: The National Natural Science Fund(81471618); Shanxi Provincial Health Department research project (201201072); Shanxi Provincial Key Research and Development Program (Social Development Field) Project (201803D31119)

Disclosure of Interests: None declared

DOI: 10.1136/annrheumdis-2019-eular.7129
$\mathrm{AB} 0210$

THE GLOBAL EXPRESSION OF MIRNAS AND LNCRNAS IN THE EXOSOMES OF SYSTEMIC SCLEROSIS PLASMA AND NEUTROPHIL AND RELATED FUNCTIONS

LI Liya, Zuo Xiaoxia, DI Liu, Luo Hui, Zhu Honglin. Department of Rheumatology and immunology, Xiangya Hospital, Central South University, Changsha, China

Background: Systemic sclerosis (SSc) is a systemic autoimmune disease with unknown pathogenesis. Exosomes (EXOs) are cell-derived vesicles 30-150 nm in size that contain various mRNAs, microRNAs (miRNAs), long non-coding RNAs (IncRNAs) and proteins. Plasma EXOs play wide roles in various diseased, however, little is known in SSc.

Objectives: Investigate the global expression of miRNAs and IncRNAs in the EXOs of SSc plasma and neutrophil. Explore the potential function of EXOs in the pathogenesis of SSc.

Methods: EXOs were respectively isolated from plasma, cultured neutrophil supernatants, and were identified by transmission electron microscopy Global expression of miRNAs and IncRNAs form $5 \mathrm{SSc}$ and 5 norma controls was analysis by IlluminaHiSeq 3000 platform. Differentiated expressed and bioinformatics analysis was performed by edgeR, limma package, GO, KEGG, and cytoscape. Furthermore, we used the EXOs stimulated human dermal microvascular endothelial cells (HDMECs) and human primary skin fibroblasts, and explore the potential functions of EXOs.

Results: 1. In plasma EXOs, we identified a total of 37 miRNAs and 479 IncRNAs that showed significant differences between the two groups. Among them, 26 of upregulated miRNAs were involved in the ErbB sig nalling pathway; 11 of downregulated miRNAs were involved in the MAPK signalling pathway, TGF- $\beta$ signalling pathway, AMPK signalling pathway;299 of upregulated IncRNAs were involved in the PI3K-Akt signalling pathway, p53 signalling pathway;180 of downregulated IncRNAs were involved in lysosome.

2. In neutrophil EXOs, we identified a total of 22 miRNAs and 281 IncRNAs that showed significant differences between the two groups Among them, 12 of upregulated miRNAs were involved in the Wnt signalling pathway; 10 of downregulated miRNAs were involved in the MAPK signalling pathway, AMPK signalling pathway; 119 of upregulated IncRNAs were involved in interleukin-23 signalling pathway;162 of downregulated IncRNAs were involved in the signalling by GPCR, signalling by $\mathrm{NOTCH}$ and TRAIL.

3. After stimulated with SSc plasma EXOs, the expression levels of has miR-324-5p and SP1/SMAD2, has-miR-624-5p and PIK3RCB, has-miR 483-5p and MAPK1 were negatively correlated, the expression levels of ENST00000562409.1 and OSMR, ENST00000601511.1 and HMGB1/NRAS were positively correlated, variably in HDMECs and human primary skin fibroblasts.

4. After stimulated with SSc neutrophil EXOs, the expression levels of has-miR-1268a and PRKCA, has-miR-299-3p and IGF1R were negatively correlated, the expression levels of ENST00000520562.1 and IL23R, ENST00000596567.1 and TFDP2, ENST00000608572.1 and HDAC2 were positively correlated, variably in neutrophil, HDMECs and human primary skin fibroblasts.

5. These miRNAs and IncRNAs with consistent expression might be involved in the pathogenesis of SSc EXOs.

Conclusion: Our study identified and confirmed differentially miRNAs and IncRNAs in the neutrophil EXOs and plasma EXOs. Those genes may be involved in the pathological mechanism of SSc, such as has-miR-324 $5 p$ and SP1/SMAD2, has-miR-624-5p and PIK3RCB, has-miR-483-5p and MAPK1, ENST00000520562.1 and IL23R, ENST00000596567.1 and TFDP2, ENST00000608572.1 and HDAC2.

Acknowledgement: This study was funded by grants from the National Key Research and Development Program of China (2016YFC0903900), National Natural Science Foundation of China (81671622), Hunan Provincial Natural Science Foundation (2018JJ3823), Clinical Research Fund of Xiangya Hospital Central South University (2014L10) and Independent Innovation Projects of Central South University (2018zzst290).

Disclosure of Interests: None declared

DOI: 10.1136/annrheumdis-2019-eular.6495 